\title{
Modeling immunotherapy of the tumor - immune interaction
}

\author{
Denise Kirschner ${ }^{1}$, John Carl Panetta ${ }^{2, *}$ \\ ${ }^{1}$ Department of Microbiology and Immunology, The University of Michigan Medical \\ School, Ann Arbor, MI 48109-0620, USA \\ ${ }^{2}$ School of Science, Penn State Erie, The Behrend College, Station Road, Erie, \\ PA 16563-0203, USA. \\ e-mail: panetta@wagner.bd.psu.edu
}

Received: 22 October 1997/Revised version: 27 November 1997

\begin{abstract}
A number of lines of evidence suggest that immunotherapy with the cytokine interleukin-2 (IL-2) may boost the immune system to fight tumors. $\mathrm{CD}^{+} \mathrm{T}$ cells, the cells that orchestrate the immune response, use these cytokines as signaling mechanisms for immune-response stimulation as well as lymphocyte stimulation, growth, and differentiation. Because tumor cells begin as 'self', the immune system may not respond in an effective way to eradicate them. Adoptive cellular immunotherapy can potentially restore or enhance these effects. We illustrate through mathematical modeling the dynamics between tumor cells, immune-effector cells, and IL-2. These efforts are able to explain both short tumor oscillations in tumor sizes as well as long-term tumor relapse. We then explore the effects of adoptive cellular immunotherapy on the model and describe under what circumstances the tumor can be eliminated.
\end{abstract}

Key words: Immunotherapy - Tumor - Cytokine - Modeling - Interleukin-2 Ordinary differential equations

\section{Introduction}

Cancer is still a leading cause of death in the world yet much is still not known about its mechanisms of establishment and destruction. While surgery and/or chemo- and radiotherapies have played key roles in treatment, it is clear that in many cases they do not represent a cure. Even when patients experience tumor regression, later relapse can occur. The need to address not only preventative measures, but also more successful treatment strategies is clear. Efforts along these lines are now being investigated through immunotherapy $[4,10,11,14,15,23,24,29-33]$. Immunotherapy refers to the use of cytokines usually together with adoptive cellular immunotherapy (ACI).

* Corresponding author 
Cytokines are protein hormones that mediate both natural and specific immunity. They are produced mainly by activated $\mathrm{T}$ cells (lymphocytes) during cellular-mediated immunity. Interleukin-2 (IL-2) is the main cytokine responsible for lymphocyte activation, growth and differentiation. It is produced by $\mathrm{CD}^{+}{ }^{+} \mathrm{T}$ cells, and in lesser quantities by $\mathrm{CD} 8^{+} \mathrm{T}$ cells (cytotoxic T cells or CTLs). IL-2 acts on the same cells that produce it. Therefore it is referred to as an autocrine growth factor although it can also act on nearby $\mathrm{T}$ lymphocytes (hence a paracrine growth factor). Clinical trials have shown that there are immune-stimulation effects from treatment with interleukins [4, $10,11,14,15]$. IL-2 has been shown to enhance CTL activity at different disease stages [29-31, 33]. Also, there is a restoration of defective natural killer (NK) cell activity as well as enhancement of polyclonal expansion of $\mathrm{CD}^{+}{ }^{+}$and $\mathrm{CD}^{+} \mathrm{T}$ cells $[32,35]$.

ACI refers to the injection of cultured immune cells that have anti-tumor reactivity into tumor bearing host. This is usually done in conjunction with large amounts of IL-2. This can take two approaches:

1. LAK-(lymphokine-activated killer cell) therapy: These cells are derived from the in vitro culturing with high concentrations of IL-2 of peripheral blood leukocytes removed from patients. The LAKs are then injected back at the cancer site. These are thought to be mainly natural killer cells.

2. TIL-(tumor infiltrating lymphocyte) therapy: These cells are derived from lymphocytes recovered from the patient tumors. They are then incubated with high concentrations of IL-2 in vitro and are comprised of activated NK cells and CTL cells. They are then injected back into the patient at the tumor site.

The theoretical study of tumor-immune dynamics has a long history. A good summary can be found in Adam and Bellomo [2]. We attempt to add to the existing literature by exploring the role of cytokines in the disease dynamics as well as address the topics of long-term tumor recurrence and short-term tumor oscillations. Presently, there is an ongoing search for more efficacious and less toxic results from treatment with immunotherapy [33]. This study aides in addressing this issue. In this paper we will formulate a model for tumor-immune dynamics (Sect. 2), explore the analysis of the model for mathematical and biological implications (Sect. 3), and in Sect. 4, we enhance the model to explore immunotherapy treatment.

\section{Model}

To begin a model of tumor-immune dynamics, we first examine some existing models. Kuznetsov et al. [18] define an ordinary differential equation (ODE) model for two main populations: effector cells and tumor cells. They predict a threshold above which there is uncontrollable tumor growth, and below which the disease is attenuated with periodic exacerbations occurring every 3-4 months. They also show the model does have stable spirals, but the 
Dulac-Bendixson criterion demonstrates there are no stable closed orbits. In DeLisi and Rescigno [6] and Adam [1], they again consider ODE's for the populations of immune and tumor cells. They show that survival increases if the immune system is stimulated. They also show in some cases that an increase in effector cells increases the chance of tumor survival. Furthermore, they give a threshold for the chance of uncontrolled tumor growth. The probability will increase if the effector-cell death rate or growth rate of the tumor cells increase, or if the growth rate of effector cells or the killing of tumor cells decreases. Nani and Oǧuztöreli [28] developed a model of ACI based on work by Rosenberg et al. [30,31]. Their model incorporates stochasticity effects on the immune-cancer interactions. Results of their model are that success of treatment is dependent on the initial tumor burden. Also, simulation of immunotherapy treatment indicated that more aggressively growing tumors will overpower the ACI treatment. They do not consider sensitivity, bifuraction, or stability analysis of the model. Finally, there is a very detailed model given by DeBoer et al. [5] with 10 (plus) differential equations coupled with $3-5$ algebraic equations describing most of the players in the tumor-immune dynamics. They are able to show both tumor regression (with a highly antigenic tumor) and uncontrolled tumor growth (for a low antigenic tumor).

Our goal is to use some of the best ideas in these systems, but to keep the model as simple as possible while incorporating the most important concepts of tumor-immune dynamics together with the feature of IL-2 dynamics. Therefore, we define three populations. These include: $E(t)$, the activated immune-system cells (commonly called effector cells) such as cytotoxic T-cells, macrophages, and natural killer cells that are cytotoxic to the tumor cells; $T(t)$, the tumor cells; and $I_{L}(t)$, the concentration of IL-2 in the single tumor-site compartment we are modeling. Our model describing the interaction between the effector cells, tumor cells, and the cytokine (IL-2) is:

$$
\begin{aligned}
& \frac{d E}{d t}=c T-\mu_{2} E+\frac{p_{1} E I_{L}}{g_{1}+I_{L}}+s_{1}, \\
& \frac{d T}{d t}=r_{2}(T) T-\frac{a E T}{g_{2}+T}, \\
& \frac{d I_{L}}{d t}=\frac{p_{2} E T}{g_{3}+T}-\mu_{3} I_{L}+s_{2},
\end{aligned}
$$

with initial conditions:

$$
E(0)=E_{0}, \quad T(0)=T_{0}, \quad I_{L}(0)=I_{L_{0}},
$$

and where the model terms are described as follows. The first equation describes the rate of change for the effector-cell population. Effector cells are stimulated to grow based on two terms. One is a recruitment term (term 1) due to the direct presence of the tumor, where the parameter $c$ models the antigenicity of the tumor. Antigenicity can be thought of as a measure of how 
different the tumor is from 'self'. The other growth/source term (term 3) is a proliferation term whereby effector cells are stimulated by IL-2 that is produced by effector cells in both an autocrine and paracrine manner. This term is of Michaelis-Menten form to indicate the saturated effects of the immune response. Effector cells have a natural lifespan of an average $1 / \mu_{2}$ days. Lastly, $s_{1}$ is a treatment term that represents an external source of effector cells such as LAK or TIL cells. Equation (2) marks the rate of change of the tumor cells. This can be described by a linear growth term (if $r_{2}(T)$ is constant) or as a type of limiting-growth such as logistic or Gompertz. We chose the logistic growth function

$$
r_{2}(T)=r_{2}(1-b T) .
$$

The loss of tumor cells is represented by an immune-effector cell interaction at rate $a$. This rate constant, $a$ represents the strength of the immune response and is modeled by Michaelis-Menten kinetics to indicate the limited immune response to the tumor. (This form could also account for the effects of a solid tumor, i.e. only a portion of the tumor mass comes in contact with the immune system cells [6].) Equation (3) gives the rate of change for the concentration of IL-2. Its source is the effector cells that are stimulated by interaction with the tumor and also has Michaelis-Menten kinetics to account for the self-limiting production of IL-2. The next term $\left(\mu_{3}\right)$ represents loss/degraded rate of IL-2. Finally, $s_{2}$ is a treatment term that represents an external input of IL-2 into the system.

\subsection{Parameter estimation}

To complete the development of the mathematical model, (1)-(5), we must define values for the parameters and initial conditions.

As terms in our model are somewhat similar in the first two equations (1) -(2) to that of the models in [5] and [18], we explored the choices for parameters as presented in those studies. In many cases there were large ranges in the parameter choices between studies and we chose values most appropriate for this model. The values are given in Table 1 (units are in days $^{-1}$ except for $g_{1}, g_{2}, g_{3}$, and $b$ whose units are volume). Values for the rate constants in equation (3), for which no previous study was done, were found from the current medical literature and from sensitivity analyses. For example, according to Rosenberg and Lotze [30] the half-life for IL-2 is between 30-120 min. We use the average value of this half-life for $\mu_{3}$ in our simulations. The value of $c$, defined as the antigenicity of the tumor varies greatly from

Table 1. Parameter values

\begin{tabular}{lllll}
\hline Eq. (1) & $0 \leqq c \leqq 0.05$ & $\mu_{2}=0.03$ & $p_{1}=0.1245$ & $g_{1}=2 \times 10^{7}$ \\
Eq. (2) & $g_{2}=1 \times 10^{5}$ & $r_{2}=0.18$ & $b=1 \times 10^{-9}$ & $a=1$ \\
Eq. (3) & $\mu_{3}=10$ & $p_{2}=5$ & $g_{3}=1 \times 10^{3}$ & \\
\hline
\end{tabular}


patient to patient and cancer to cancer. Larger values of $c$ represent tumor cells that present a well recognized antigen and smaller values represent tumor cells that present a weak antigen. We explore a wide range of values for $c$. After carrying out a sensitivity analysis on the parameters $c$, the tumor antigenicity, as well as $a$, the immune response, it is clear they play key roles in the dynamics. We explore these rate constant through bifurcation analyses in the next sections.

\section{Analysis and numerical results}

Equations (1)-(3), together with initials conditions (4) represent the tumorimmune model in the absence of treatment. For ease of analysis, we first non-dimensionalize the system, and then carry out a steady-state analysis on the scaled system. We also discuss the biological relevance of our results.

\subsection{Scaling}

We non-dimensionalize the model (1)-(3) using the following scaling:

$$
\begin{aligned}
& x=\frac{E}{E_{0}}, \quad y=\frac{T}{T_{0}}, \quad z=\frac{I_{L}}{I_{L_{0}}}, \quad \tau=t_{s} t, \quad \bar{c}=\frac{c T_{0}}{t_{s} E_{0}}, \\
& \bar{p}_{1}=\frac{p_{1}}{t_{s}}, \quad \bar{g}_{1}=\frac{g_{1}}{I_{L_{0}}}, \quad \bar{\mu}_{2}=\frac{\mu_{2}}{t_{s}}, \quad \bar{g}_{2}=\frac{g_{2}}{T_{0}}, \quad \bar{b}=b T_{0}, \\
& \bar{r}_{2}=\frac{r_{2}}{t_{s}}, \quad \bar{a}=\frac{a E_{0}}{t_{s} T_{0}}, \quad \bar{\mu}_{3}=\frac{\mu_{3}}{t_{s}}, \quad \bar{p}_{2}=\frac{p_{2} E_{0}}{t_{s} I_{L_{0}}}, \quad \bar{g}_{3}=\frac{g_{3}}{T_{0}}, \\
& s_{1}=\frac{s_{1}}{t_{s} E_{0}}, \quad \bar{s}_{2}=\frac{s_{2}}{t_{s} I_{L_{0}}} .
\end{aligned}
$$

Then, dropping the over-bar notation for convenience, we obtain the following scaled model:

$$
\begin{aligned}
& \frac{d x}{d \tau}=c y-\mu_{2} x+\frac{p_{1} x z}{g_{1}+z}+s_{1} \\
& \frac{d y}{d \tau}=r_{2} y(1-b y)-\frac{a x y}{g_{2}+y} \\
& \frac{d z}{d \tau}=\frac{p_{2} x y}{g_{3}+y}-\mu_{3} z+s_{2}
\end{aligned}
$$

with initial conditions:

$$
x(0)=x_{0}, \quad y(0)=y_{0}, \quad z(0)=z_{0} .
$$

One possible choice for the scaling is to define: $E_{0}=T_{0}=I_{L_{0}}=1 / b$ and $t_{s}=r_{2}$. These scalings need to be chosen to help adjust for the fact that this is 


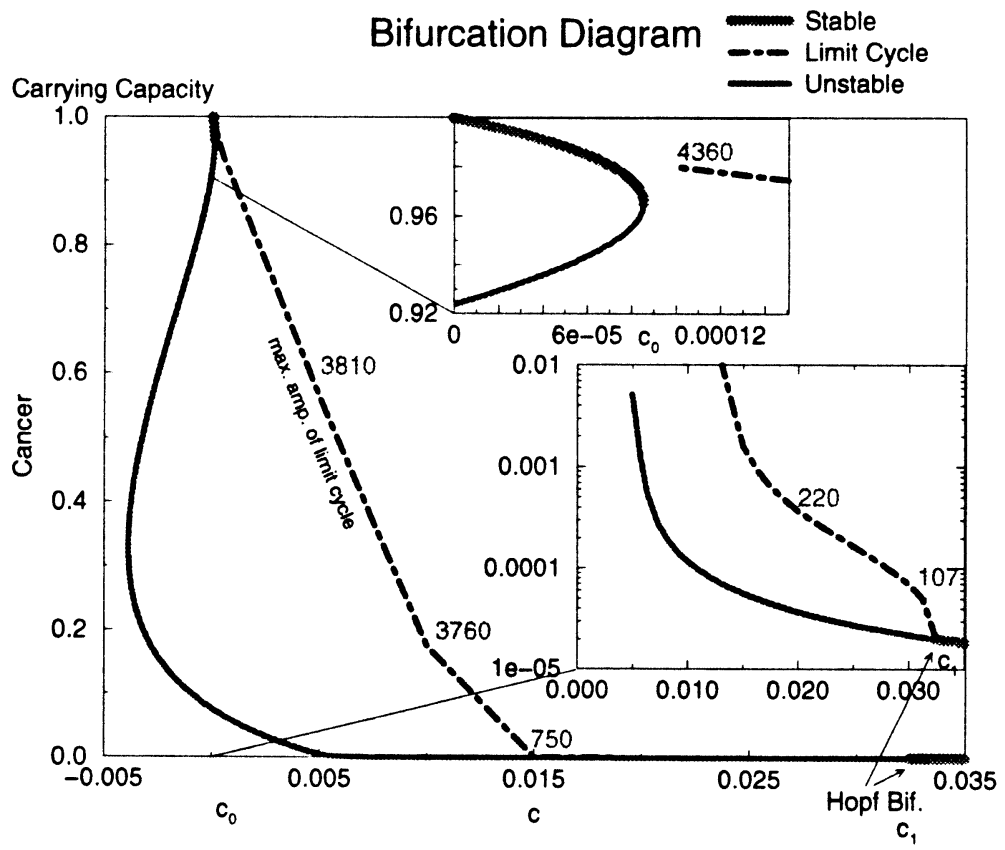

Fig. 1. A bifurcation diagram varying the antigenicity $(c)$. The numbers along the maximal amplitude limit-cycle curve represent the corresponding period of the limit cycle in days. The carrying capacity of the tumor is scaled to 1

a numerically "stiff" system. That is, without scaling, or inappropriate scalings, the numerical routines used to solve these equations will fail. This is due to very large changes in some of the variables over very short ranges of time.

\subsection{Stability analysis - no treatment case}

The dynamics of this model (6)-(9) are very rich. We begin by exploring the steady-states when there are no treatment terms, i.e. both $s_{1}$ and $s_{2}$ are zero. The first equilibrium is the trivial state where all the populations are zero, namely $E_{0}=(0,0,0)$. The eigenvalues of the Jacobian matrix for $E_{0}$ are $-\mu_{2}$, $r_{2}$, and $-\mu_{3}$. Therefore, $E_{0}$ is always a locally unstable saddle point. There exists multiple, positive, non-trivial steady states, depending on the choice of parameters, namely $E_{i}=\left(E_{i}^{*}, T_{i}^{*}, I_{L_{i}}^{*}\right)$ where $i$ can range from 1 to 3 . To aid in explaining these different outcomes, we present a bifurcation diagram ${ }^{1}$ for the parameter $c$, the tumor antigenicity (see Fig. 1). There are three categories for the dynamics as $c$ is varied. These can each be seen in Figs. 1 and 2 .

\footnotetext{
${ }^{1}$ All the numerical bifurcation analysis in this and the following sections was carried out using "XPPAUT3.0" by Ermentrout [8] that incorporates the bifurcation analysis program "AUTO" by Doedel [7]
} 
A

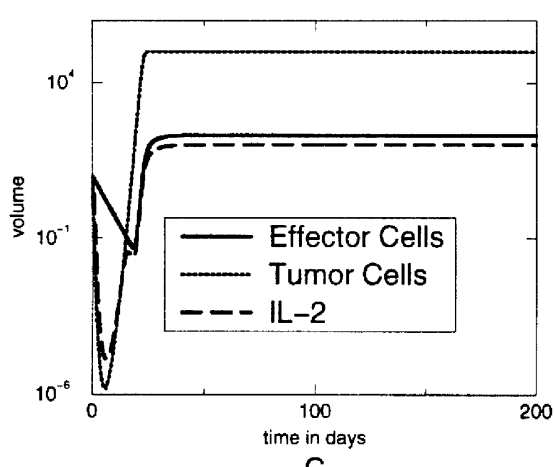

C

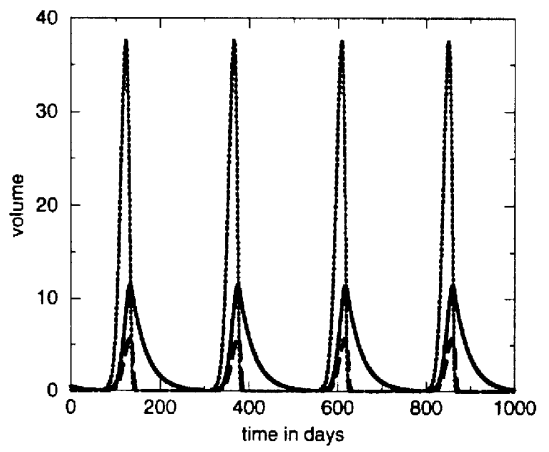

B

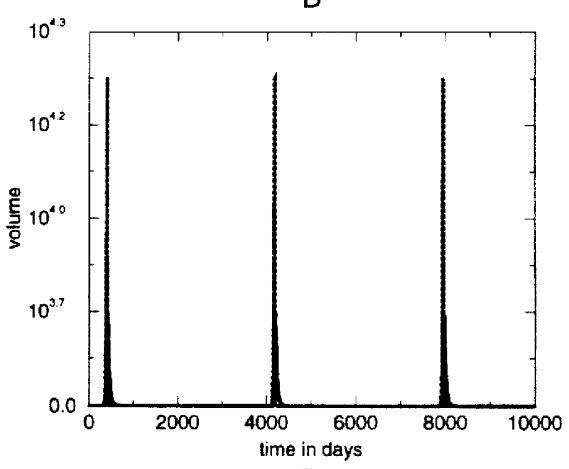

D

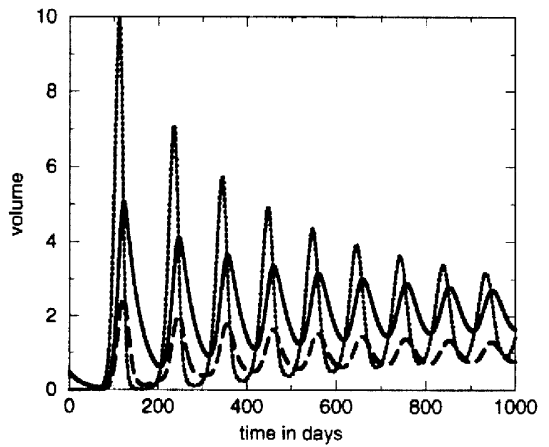

Fig. 2. Effector cells (solid lines), tumor cells (dotted lines), and IL-2 (long dashed lines) vs. time. Time is in days. The carrying capacity of the tumor is scaled to $1 \mathrm{e}+5$. (A) $c=5 e-5$, (B) $c=0.01$, (C) $c=0.02$, (D) $c=0.035$

1. Region $0<c<c_{0}$ (Insert at top of Fig. 1 and Fig. 2A. Note that $c_{0}=8.55 \times 10^{-5}$ for the parameter values listed in Table 1.) In this region there is one stable, positive steady state and two unstable, positive steady states. The steady-state $\left(E_{1}\right)$ is a locally stable, improper node such that the tumor is very large, almost at the size of its carrying capacity $(1 / b)$. The unstable steady-states $\left(E_{2}\right.$ and $\left.E_{3}\right)$ are respectively, an unstable spiral that persists until $c=c_{1}$, and an unstable saddle.

2. Region $c_{0}<c<c_{1}$. (Main Fig. 1 and Figs. 2B and C. Note that $c_{1}=0.032$ for the parameter values listed in Table 1.) At $c=c_{0}$, the states $E_{1}$ and $E_{3}$ are lost but a stable limit cycle is 'born'. This cycle persists for the entire interval, but as the value of $c$ increases towards $c_{1}$ (the Hopf bifurcation) the amplitude and period of the limit cycles decreases. At a value close to $c_{0}$ (e.g. $c=0.001$ ) near where the limit cycle is born, the period of the limit cycle is 3900 days (using the parameters in Table 1) and the cancer is detectable (cancer mass near $90 \%$ of carrying capacity $1 / b$ ) for about 100 days. However, for a larger choice of $c$ (e.g. $c=0.01)$ the period of the limit cycle is 3250 days and the cancer mass is detectable for about 45 days reaching a maximum mass of about $10 \%$ of the carrying capacity of the 
cancer. For values of $c$ close to $c_{1}$ (e.g. $c=0.031$ ) the period of the limit cycle is 107 days, with a very small maximum tumor size. The steady state $E_{2}$ is still present, and unstable.

3. Region $c_{1}<c$ (Insert at bottom of Fig. 1 and Fig. 2D.) At $c_{1}$, the state $E_{2}$ bifurcates from an unstable, to a stable, spiral node via a Hopf bifurcation. The oscillations are small and damp out quickly. The masses of the tumors in this region are small (about 5 orders of magnitude less than that of the $E_{1}$ size for $c<c_{1}$ ).

\subsection{Biological implications}

The implications of these bifurcation results are provocative. In case 1, the model allows for the possibility of a large tumor mass when the tumor antigenicity, $c$, is extremely small. Case 2 , however, presents a very different outcome. It implies that for a very low antigenic tumor (small $c$ ), the tumor mass cycles with a large period (as long as 11 years). The tumor will spend a portion of the cycle (as much as 2 or 3 months) near its carrying capacity and then the remainder of the 11 years with a mass near zero, i.e. dormant. (A small stable tumor that does not change in size is referred to as dormant). This could explain long term recurrence of tumors within-host. There is clinical evidence to support this recurrent phenomenon $[3,12,13,34]$. Also, Tsao et al. [36] has found recurrence of melanoma more than 15 years after the patients initial contact with the disease. As the tumor antigenicity increases, the magnitude and period of these oscillations shorten, as well as the length of time the tumor remains at its peak size. This progresses until the oscillations decrease down to a monthly basis and the tumor size is very small with a quick recovery time. There is experimental evidence of these short term oscillations in cases like Chronic Myeloid Leukemia as well as others [9, 16, 17, 25, 37]. Also Gause et al. [10] observe short-term oscillations in the lymphocyte counts with a monthly period. Finally, these oscillations give way to a stable spiral with very quick damping, leading to a small, persistent tumor, that could be described as dormant.

A natural question that arises from the above study is, what affects the period of these periodic solutions? We are thus interested in how key parameters, namely antigenicity $(c)$ and immune response $(a)$, affect the period of the limit cycles discussed above; and we present a 2-parameter bifurcation diagram. In particular, Fig. 3 shows how the parameters $a$ and $c$ affect the period of the limit cycle. Each curve in Fig. 3 represents a fixed period of the limit cycle. Note that for fixed $c$, an increase in $a$ (the immune response) causes a decrease in the period (and thus the amplitude) of the limit cycle. If $a$ is increased enough then we pass through the Hopf Bifurcation and the limit cycle disappears. In this case the tumor volume is small and dormant.

In the non-treatment case (i.e. $s_{1}=s_{2}=0$ ), the model does not allow for complete clearance of the tumor. To this end, we now study the role of enhancing the immune response through immunotherapy. 


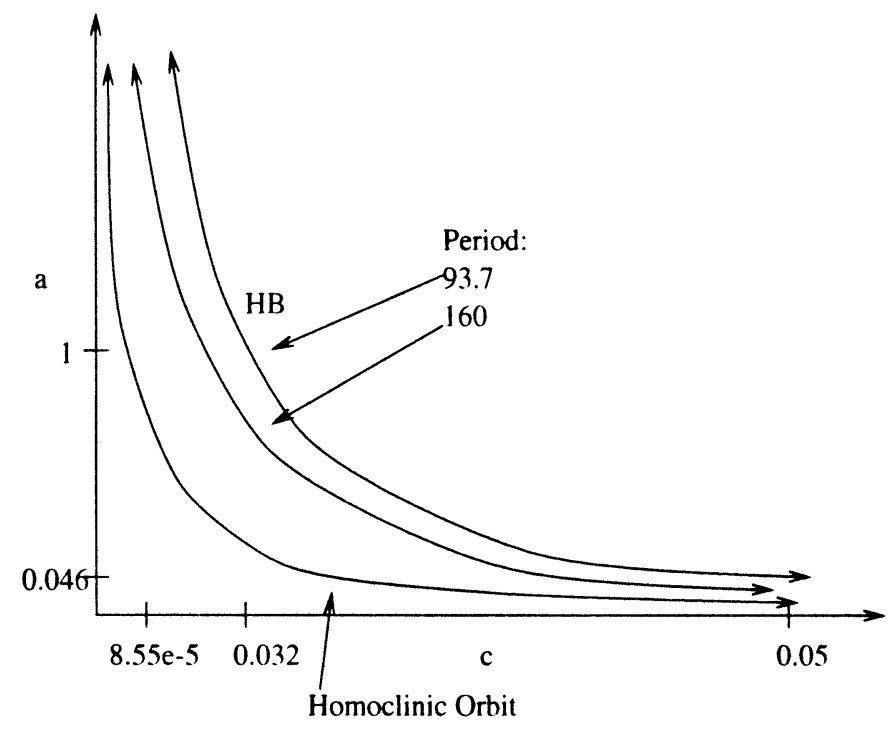

Fig. 3. Fixed period curves of antigenicity vs. the immune response ( $c$ vs $a$ ). The period of the limit cycles are constant for values of $a$ and $c$ on each curve. HB $=$ Hopf Bifurcation curve

\section{Immunotherapy}

To date, most treatment of cancer focuses on chemo- and radiotherapies directed at the tumor. Here we explore treatment that serves to boost the immune-system's capacity to fight the cancer. Immunotherapy attempts to use cytokines, the communication/stimulation proteins produced, released, and used by cells, to enhance cellular activity. The cytokine most effective in this regard is interleukin-2, as it is the key interleukin responsible for $\mathrm{T}$-cell growth and differentiation; the cells that orchestrate the immune response. We will consider immunotherapy to be adoptive cellular immunotherapy and/or IL-2 delivery at the tumor site either separately or in combination. Thus, in model (1)-(4) we consider either or both $s_{1}$ and $s_{2}$ to be non-zero where rate $s_{1}$ represents the addition of LAK or TIL cells to the tumor site and rate $s_{2}$ represents the addition of interleukin-2 to the site. We explore each of these therapies separately and then the combined effects of both.

\subsection{Adoptive cellular immunotherapy $\left(s_{1}>0, s_{2}=0\right)$}

In the absence of $s_{1}$ the trivial non-cancer steady state, $E_{0}$, is always unstable. In the presence of $s_{1}$ this state is lost, but there is another state that is a more 
realistic non-tumor state, $E_{0}=\left(E_{0}^{*}, 0,0\right)$. This implies that effector cells can clear the tumor if this equilibrium is stable. From analyzing the eigenvalues, this state is locally stable if $s_{1}>s_{\text {crit }}^{1}$ and unstable if $s_{1}<s_{\text {crit }}^{1}$ where

$$
s_{\text {crit }}^{1}=\frac{r_{2} g_{2} \mu_{2}}{a} .
$$

(Note that the value of $s_{\text {crit }}^{1}=540$ for the parameter values in Table 1.) Hence, when $s_{1}=s_{\text {crit }}^{1}$ there is a bifurcation that, with the analysis to follow, can be shown to be a transcritical bifurcation.

In the cases where a tumor can exist the dynamics are again very rich. Here, the positive steady states are of the form $E_{i}=\left(E_{i}^{*}, T_{i}^{*}, I_{L_{i}}^{*}\right), i$ ranging from 1 to 3 . These vary with the treatment input $s_{1}$. We examine regions in the $c$ vs $s_{1}$ parameter space to explore how $s_{1}>0$ alters the earlier results. The Roman numerals (below) correspond to the regions of the bifurcation diagram in Fig. 4.

A. If $s_{1}<s_{\text {crit }}^{1}$ :

I. There is only one steady-state, $E_{1}=\left(E_{1}^{*}, T_{1}^{*}, 0\right)$, and it is a stable improper node.

II. A stable limit cycle is born. These cycles persist for the entire region, but as the parameters approach the value of the Hopf Bifurcation, the amplitude and the period of the limit cycles decreases.

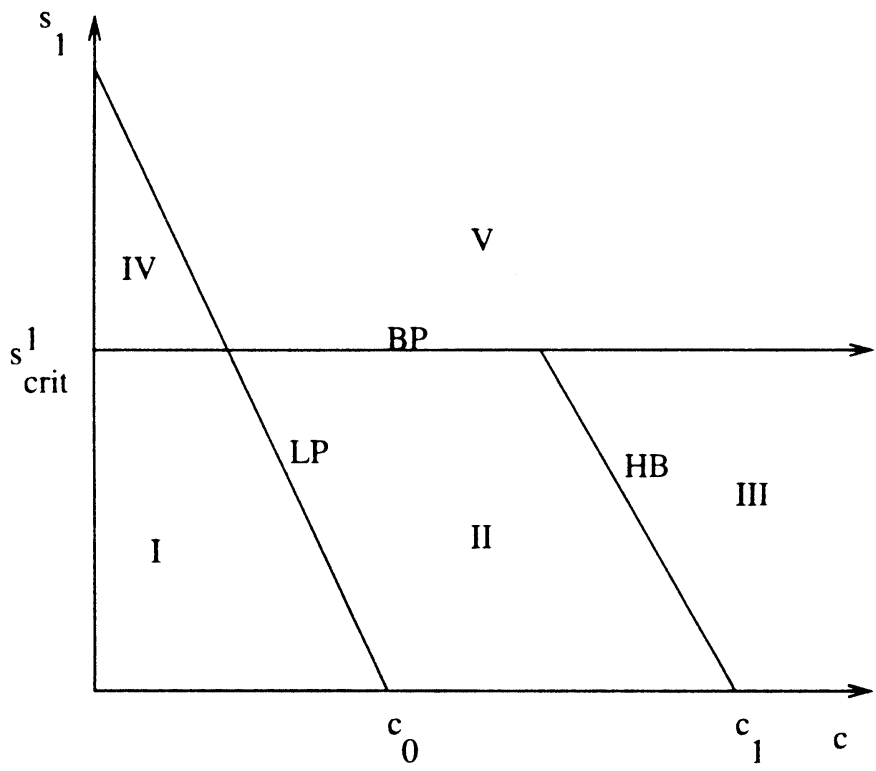

Fig. 4. A two-parameter bifurcation diagram of antigenicity versus ACI ( $c$ vs. $s_{1}$ ). $\mathrm{HB}=$ Hopf Bifurcation; LP $=$ Limit Point; $\mathrm{BP}=$ Bifurcation Point. The regions are described in the text 
III. There is one steady state $E_{3}=\left(E_{3}^{*}, T_{3}^{*}, I_{L_{3}}^{*}\right)$ that is born as a result of a Hopf bifurcation. It is a stable spiral node.

B. If $s_{1}>s_{\text {crit }}^{1}$ :

IV. This is a region of bistability. Depending on the initial condition we start with either the solution will tend either to $E_{1}$ (the tumor survives) or to $E_{0}$ (the immune system succeeds). This phenomenon of bistability has been previously studied with regard to tumor immunology $[20,21]$,

V. In this region $E_{0}$ is stable. Thus, the immune system succeeds in clearing the tumor. Note that this is the largest area of parameter space.

\subsection{Interleukin-2 $\left(s_{1}=0, s_{2}>0\right)$}

We now explore the input of IL-2 into the system. The only non-tumor state is $E_{0}=\left(0,0, \frac{S_{2}}{\mu_{3}}\right)$. This state is always an unstable saddle point. This may imply that administering IL-2 alone, without ACI, cannot clear the tumor. If $s_{2}$ is small, we expect the dynamics to be similar to that of the untreated case. If $s_{2}$ is large,

$$
s_{2}>\frac{\mu_{2} \mu_{3} g_{1}}{p_{1}-\mu_{2}}=s_{c r i t}^{2}
$$

then the effector cells will grow uncontrolled and the only stable 'state' is $\left(\infty, 0, s_{2} / \mu_{3}\right)$. To see this we must show that $d E / d t>0$ when (11) holds. Plugging $I_{L}=s_{2} / \mu_{3}$ into Eq. (1) and simplifying we find:

$$
\frac{d E}{d t}=E\left(\frac{\left(p_{1}-\mu_{2}\right) s_{2}-\mu_{2} \mu_{3} g_{1}}{s_{2}+\mu_{3} g_{1}}\right) .
$$

Therefore, for $d E / d t>0$, the numerator of equation (12) must be greater than zero; this is equivalent to equation (11). Thus, if (11) holds then $\left(\infty, 0, s_{2} / \mu_{3}\right)$ is stable. The bifurcation diagram in Fig. 5 shows the different dynamics involved with varying $s_{2}$ with respect to $c$. The regions of Fig. 5 are described as follows, with the biological relevance presented in Sect. 4.4 (Again, note that the Roman numerals below correspond to those in Fig. 5):

I. The cancer grows to near its carrying capacity. In this case both the antigenicity and the IL-2 concentration is small, so the result parallels the non-treatment case.

II. Stable limit cycles exist (as in Fig. 4).

III. Stable spirals leading to a stable, small tumor (as in Fig. 4).

IV. $\left(\infty, 0, s_{2} / \mu_{3}\right)$ is the only stable 'equilibria'. This state is a new dynamic acquired from the introduction of the non-zero constant source, $s_{2}$, in equation (3). 


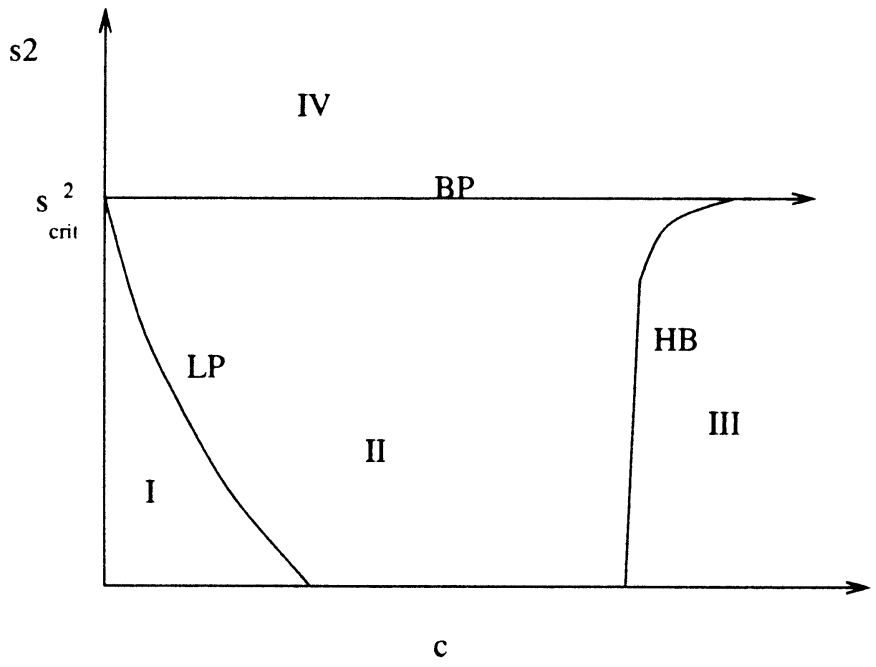

Fig. 5. Two-parameter bifurcation diagram of antigenicity versus IL-2 ( $c$ vs $\left.s_{2}\right)$. HB $=$ Hopf Bifurcation; LP $=$ Limit Point; $\mathrm{BP}=$ Bifurcation Point. The regions are described in the text

\subsection{Immunotherapy with both ACI and IL-2 ( $\left.s_{1}>0, s_{2}>0\right)$}

In this case we have both treatment terms non-zero in (6)-(8). The tumor free equilibrium is now $E_{0}=\left(E_{0}^{*}, 0, I_{L 0}^{*}\right)$ where

$$
\begin{aligned}
& E_{0}^{*}=\frac{s_{1}\left(\mu_{3} g_{1}+s_{2}\right)}{\mu_{2}\left(\mu_{3} g_{1}+s_{2}\right)-s_{2} p_{1}}, \\
& I_{L 0}^{*}=\frac{s_{2}}{\mu_{3}} .
\end{aligned}
$$

The eigenvalues of the Jacobian matrix are:

$$
\frac{p_{1} I_{L 0}^{*}}{g_{1}+I_{L 0}^{*}}-\mu_{2}, \quad r_{2}-\frac{a E_{0}^{*}}{g_{2}}, \quad-\mu_{3} .
$$

Therefore, for this equilibria to be locally stable we require

$$
s_{2}<\frac{\mu_{2} \mu_{3} g_{1}}{p_{1}-\mu_{2}}=s_{c r i t}^{2}
$$

and

$$
s_{1}>\frac{g_{2} r_{2}}{a}\left[\frac{s_{2}\left(\mu_{2}-p_{1}\right)+\mu_{2} \mu_{3} g_{1}}{\mu_{3} g_{1}+s_{2}}\right] .
$$

Note that for the parameters given in Table $1, s_{\text {crit }}^{2}=63492063$. Varying the antigenicity $(c)$ affects the $s_{1}$ vs $s_{2}$ bifurcation diagram. Table 2 and 
Table 2. Summary of results for Fig. 6. KEY: $\mathrm{CF}=$ cancer free state; $\mathrm{CCC}=$ Cancer grows to carrying capacity $\left(E_{3}\right) ; \mathrm{LC}=$ Limit cycles are present; N/A not applicable; $\mathrm{CFI}=$ cancer free with immune system growing without bounds to $\left(\infty, 0, I_{L 0}^{*}\right)$-stable; SCSS $=$ small cancer state from a stable spiral; $\mathrm{BS}=$ Bistability to either $\mathrm{CF}$ or $\mathrm{CCC}$

\begin{tabular}{llllll}
\hline & Figure & Region I & Region II & Region III & Region IV \\
\hline$c=0$ & 5A & CCC & BS & CF & CFI \\
$c=8.5 e^{-5}$ & 5B & CCC & LC & CF & CFI \\
$c=0.0025$ & 5C & N/A & LC & CF & CFI \\
$c=0.006$ & 5D & LC & SCSS & CF & CFI \\
$c=0.0325$ & 5E & LC & SCSS & CF & CFI \\
$c=0.04$ & 5F & N/A & SCSS & CF & CFI \\
\hline
\end{tabular}

Fig. 6 summarize the results. The biological relevance is discussed below in section (4.4).

\subsection{Biological significance}

The results in Sects. 4.1, 4.2, and 4.3 again present some interesting biological interpretations. For the treatment results, the most significant correlations are between antigenicity of the tumor together with the type of treatment being administered. We focus our discussion on those correlations in detail.

In Sect. 4.1 the two key concepts explored are the antigenicity $(c)$ and the administration of adoptive cellular immunity $\left(s_{1}\right)$. The bifurcation diagram in Fig. 1 for antigenicity, $c$, is indicative of the same general effects of ACI treatment for different tumors shown in Fig. 4. For low antigenic tumors and a low input of treatment, the tumor remains large and stable. For low antigenic tumors but larger amounts of treatment bistability exists where either the large-tumor state or the tumor-free state accepts stability. If the treatment is increased to very high levels then the tumor can be cleared. For tumors that exhibit a medium to high antigenicity there are two cases. If little immunotherapy is input into the system, then the situation remains basically the same as with no treatment. But, with medium to high immunotherapy, the tumor can be cleared.

In Sect. 4.2, the two key concepts we explored are the antigenicity $(c)$ and the administration of IL-2 $\left(s_{2}\right)$. The bifurcation diagram in Fig. 5 reveals the comparative effects. Administering low concentrations of IL-2 (below $s_{\text {crit }}^{2}$ ) yields qualitatively the same results as with no IL-2 treatment (see Fig. 1). However, large amounts of administrated IL-2 (above $s_{\text {crit }}^{2}$ ) together with any degree of antigenicity yields an interesting result. Here, the tumor is cleared but the immune system grows unbounded as the IL-2 concentration reaches a steady-state value. This uncontrolled growth of the immune system represents a situation that is detrimental to the host. A well-documented side effect from treatment with dose escalations of IL-2 is capillary leak syndrome (or vascular leakage syndrome) $[19,22,26,32]$. This syndrome has been 

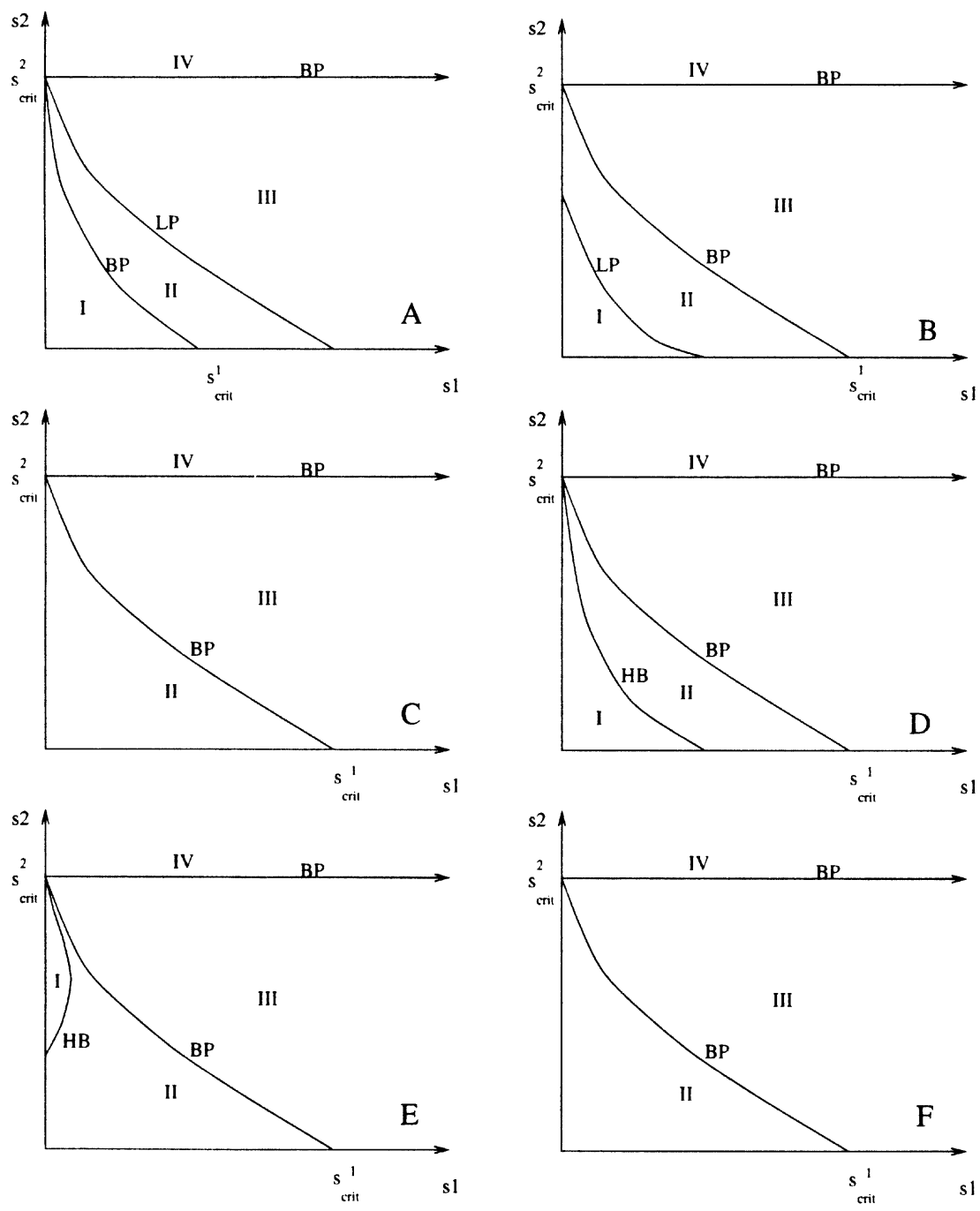

Fig. 6. Two-parameter bifurcation diagram of IL-2 versus ACI $\left(s_{1}\right.$ vs $\left.s_{2}\right)$ for different $\begin{array}{llll}\text { antigenicity levels: } & \text { (A) } c=0 . & \text { (B) } c=8.5 e-5 . & \text { (C) } c=0.0025\end{array} \quad$ (D) $c=0.006$. (E) $c=0.0325$. (F) $c=0.04$. HB $=$ Hopf Bifurcation; $\mathrm{LP}=$ Limit Point; $\mathrm{BP}=$ Bifurcation Point. The regions are described in the text

attributed to a variety of IL-2-induced activation events [22, 32]. This could imply that for values of IL-2 administered above $s_{\text {crit }}^{2}$ the unbounded growth of the immune effector cells may indeed reflect the detrimental side effects seen in cancer patients receiving immunotherapy.

In section (4.3) we combine the treatment strategies of ACI and IL-2. A summary of the effects of simultaneous treatments is presented in Table 2 
and Fig. 6. The results are a combination of the separate mono-treatment regimens. For concentrations of IL-2 administered below $s_{\text {crit }}^{2}$, the effects of administering ACI concurrently make a difference for tumor clearance; and, the greater the antigenicity of the tumor the more likely the treatment will succeed. For values of IL-2 administered above $s_{\text {crit }}^{2}$ the tumor can be cleared, but the side effects from an over-activated immune system may out way the benefits of tumor clearance.

\section{Discussion}

In this paper we explore the effects of the cytokine interleukin-2 on tumorimmune dynamics. In these dynamics a key role is played by the antigenicity of the tumor. For low antigenic tumors the immune system is not able to clear the tumor; while, for highly antigenic tumors, reduction to a small dormant tumor is the best case scenario. An interesting intermediate result (for tumors exhibiting average antigenicity) is the presence of stable limit cycles which imply that the tumor and the immune system undergo oscillations. If the tumor has a low to medium antigenicity these cycles are relatively long (on the order of 8 to 10 years) with large amplitudes and the tumor is in a dormant state during most of the period. This may explain long term recurrence of tumors. If the tumor has medium to high antigenicity the periods of the cycles are short with small amplitudes (Figs. 1 and 2). The health of the immunesystem activation plays a role in the periodicity of these cycles (Fig. 3). For example, as the immune response improves (i.e. $a$ increases), the period and amplitude of the limit cycles decrease, leading to a dormant-tumor state. It should be noted that these stable limit cycles have not been observed in previous models of the interaction between tumors and the immune system (e.g., $[1,5,18])$.

Next we studied treatment of the tumor-immune system with immunotherapy. Two types of treatments are explored individually and together doptive cellular immunotherapy and administration of the cytokine IL-2. Unlike in the no treatment case (Section 2), the effects of ACI therapy can yield a tumor-free state. This happens when the treatment concentration is above a given critical level (Fig. 4). This is true for tumors of almost any antigenicity. But for tumors with small antigenicity bistability can occur with the tumorfree state and the near-carrying-capacity state both stable. This indicates the need for starting treatment early, while the tumor is small, so that the tumor can be controlled.

Treatment with IL-2 alone does not offer a satisfactory outcome; if IL-2 administration is low there is no tumor-free state. However, if IL-2 input is high the tumor can be cleared but the immune system grows without bounds causing problems such as capillary leak syndrome (Fig. 5). Finally, it is treatment with ACI and IL-2 that gives the combined effects obtained from the monotherapy regimes (Fig. 6). For any antigenicity, there is a region of tumor clearance. 
These results indicate that treatment with ACI may be a better option either as a monotherapy or in conjunction with IL-2. With IL-2 treatment alone, too little does not boost the immune system enough to clear the tumor; however, large amounts can have pathologic effects. IL-2 can augment the ACI treatment indicating that the combined effects may be the best-case scenario. In either case, it is clear that cytokine-enhanced immune function can play a significant role in treatment of cancer.

Natural extensions of this model are as follows. First, a more mechanisticbased method of modeling immunotherapy is needed. An improved version may include a time dependent source term for immunotherapy. Also, IL-2 is just one of a dozen cytokines involved in the cellular dynamics of the immune system response to tumor invasion. We plan to investigate how other cytokines such as Interferon- $\gamma$, IL-10, and IL-12 affect the dynamics of the system. These dynamics may include both positive and negative feedback mechanisms since, for example, IL-10 down-regulates cytokine production by activated macrophages. Finally, a model including the combined effects of immunotherapy with chemo- and/or radio-therapies is needed to better elaborate the results obtained here for the purpose of guiding clinical trials.

\section{References}

1. J. A. Adam. Effects of vascularization on lymphocyte/tumor cell dynamics: Qualitative features. Mathl. Comput. Modelling, 23(6): 1-10, 1996

2. J. A. Adam and N. Bellomo. A survey of Models for Tumor-Immune System Dynamics. Birkhäuser, Boston, MA, 1996

3. N. Blumberg, C. Chuang-Stein and J. M. Heal. The relationship of blook transfustion, tumor staging, and cancer recurrence. Transfusion, 30(4): 291-294, 1990

4. Brendan D. Curti, Augusto C. Ochoa, Walter J. Urba, W. Gregory Alvord, William C. Kopp, Gerry Powers, Connie Hawk, Stephen P. Creekmore, Barry L. Gause, John E. Janik, Jon T. Holmlund, Peter Kremers, Robert G. Fenton, Langdon Miller, Mario Sznol, John W. Smith II, William H. Sharfman and Dan L. Longo. Influence of interleukin-2 regimens on circulating populations of lymphocytes after adoptive transfer of anti-CD3-stimulated T cells: Results from a phase I trial in cancer patients. Journal of Immunotherapy, 19(4): 296-308, 1996

5. R. J. DeBoer, Pauline Hogeweg, Hub F. J. Dullens, Roel A. DeWeger and Willem DenOtter. Macrophage T Lymphocyte interactions in the anti-tumor immune response: A mathematical model. The Journal of Immunology, 134(4): 2748-2758, April 1985

6. Charles DeLisi and Aldo Rescigno. Immune surveillance and neoplasia - I: a minimal mathematical model. Bull. Math. Biol., 39: 201-221, 1977

7. E. Doedel. AUTO - continuation and bifurcation software for ordinary differential equations.doedel@cs.concordia.edu, 1981

8. G. Bard Ermentrout. XPPAUT3.0 - the differential equations tool. http://info.pitt.edu/ $\sim$ phase/, 1997

9. Richard A. Gatti, William A. Robinson, Amos S. Deinard, Mark Nesbit, Jeffrey J. McCullough, Mark Ballow and Robert A. Good. Cyclic leukocytosis in chronic myelogenous leukemia: New perspectives on pahtogenesis and therapy. Bood, 41(6): 771-781, June 1973 
10. B. L. Gause, M. Sznol, W. C. Kopp, J. E. Janik, J. W. Smith II, R. G. Steis, W. J. Urba, W. Sharfman, R. G. Fenton, S. P. Creekmore, J. Holmlund, K. C. Conlon, L. A. VanderMolen and D. L. Longo. Phase I study of subcutaneously administered interleuking- 2 in combination with interferon alfa- $2 \mathrm{a}$ in patients with advanced cancer. Journal of Clinical Oncology, 14(8): 2234-2241, August 1996

11. Isao Hara, H. Hotta, N. Sato, H. Eto, S. Arakawa and S. Kamidono. Rejection of mouse renal cell carcinoma elicited by local secretion of interleukin-2. Jpn. J. Cancer Res., 87: 724-729, 1996

12. Yoshihiko Hirao, Eigoro Okajima, Seiichiro Ozono, Shoji Samma, Kenji Sasaki, Tadashi Hiramatsu, Katsuhiro Babaya, Shuji Watanabe and Yoshio Maruyama. A prospective randomixed study of prophylaxis of tumor recurrence following transurethral resection of superficial bladder cancer-intravesical thio-TEPA versus oral UFT. Cancer Chemother Pharmacol, 30: S26-S30, 1992

13. Madelyn Holzman, Jr. C. Eugene Carlton and Peter T. Scardino. The frequency and morbidity of local tumor recurrence after definitive radiotherapy for stage c prostate cancer. The Journal of Urology, 146: 1578-1582, December 1991

14. Raymond Kaempfer, L. Gerez, H. Farbstein, L. Madar, O. Hirschman, R. Nussinovich and A. Shapiro. Prediction of response to treatment in superficial bladder carcinoma through pattern of interleukin-2 gene expression. Journal of Clinical Oncology, 14(6): 1778-1786, June 1996

15. U. Keilholz, C. Scheibenbogen, E. Stoelben, H. D. Saeger and W. Hunstein. Immunotherapy of metastatic melanoma with interferon-alpha and interleukin-2: pattern of progression in responders and patients with stable disease with or without resection of residual lesions. European Journal of Cancer, 30A(7): 955-958, 1994

16. B. J. Kennedy. Cyclic leukocyte oscillations in chronic mylegenous leukemia during hydroxyrtea therapy. Bood, 35(6): 751-760, June 1970

17. John G. Krikorian, Carol S. Portlock, D. Paul Cooney and Saul A. Rosenberg. Spontaneous regression of non-hodgkin's lymphoma: A report of nine cases. Cancer, 46: 2093-2099, 1980

18. V. A. Kuznetsov, I. A. Makalkin, M. A. Taylor and A. S. Perelson. Nonlinear dynamics of immunogenic tumors: Parameter estimation and global bifurcation analysis. Bull. Math. Biol., 56(2): 295-321, 1994

19. R. E. Lee, M. T. Lotze, J. M. Skibber, E. Tucker, R. O. Bonow, F. P. Ognibene, J. A. Carrasquillo, J. H. Shelhamer, J. E. Parillo and S. A. Rosenberg. Cardiorespiratory effects of ummunoltherapy with interleukin-2. Journal of Clinical Oncology, 7(1): 7-20, Jan 1989

20. R. Lefever and W. Horsthemke. Bistability in fluctuating environments. implications in tumor immunology. Bull. Math. Biol., 41: 469-90, 1979

21. Rene Lefever and Thomas Erneaux. On the growth of cellular tissues under constant and fluctuating environmental conditions. pp. 287-305, 1983

22. P. Lissoni, S. Barni, G. Cattaneo, C. Archili, S. Crispino, G. Tancine, L. D’Angelo, S. Magni, and G. Fiorelli. Activation of the complement system during immunotherapy of cancer with interleukin-2: a possible explanation of the capillary leak syndrome. International Journal of Biological Markers, 5(4): 195-197, Oct-Dec 1990

23. Angela J. Lumsden, James P. Codde, Peter H. Van Der Meide and Bruce N. Gray. Immunohistochemical characterisation of immunological changes at the tumour site after chemo-immunotherapy with doxorubicin, interleukin- 2 and interferon- $\gamma$. Antocancer Research, 16: 1145-1154, 1996

24. F. M. Marincola, D. E. White, A. P. Wise and S. A. Rosenberg. Combination therapy with interferon alfa-2a and interleukin-2 for the treatment of metastatic cancer. Journal of Clinical Oncology, 13(5): 1110-1122, 1996

25. B. C. Mehta and M. B. Agarwal. Cyclic oscillations in leukocyte count in chronic myeloid leukemia. Acta Haematologica, 63: 68-70, 1980.

26. D. W. Miles, L. C. Happerfield, L. G. Bobrow and R. D. Rubens. Adhesion molecule expression and leucocyte rafficking following immunotherapy with recombinant interleukin-2. Histopatholgy, 28(4): 301-308, Apr 1996 
27. A. A. Morley, A. G. Baikie, and D. A. G. Galton. Cyclic leucocytosis as evidence for retention of normal homoeostatic control in chronic granulocytic leukaemia. The Lancet, 1320-1323, December 1967

28. F. K. Nani and M. N. Oğuztöreli. Modelling and simulation of Rosenberg-type adoptive cellular immunotherapy. IMA Journal of Mathematics Applied in Medicine \& Biology, 11: 107-147, 1994

29. Hannah Rabinowich, Michael Banks, Torten E. Reichert, Theodore F. Logan, John M. Kirkwood and Theresa L. Whiteside. Expression and activity of signaling molecules $\mathrm{n} \mathrm{T}$ lymphocytes obtained from patients with metastatic melanoma before and after interleukin 2 therapy. Clinical Cancer Research, 2: 1263-1274, 1996.

30. S. A. Rosenberg and M. T. Lotze. Cancer immunotherapy using interleukin-2 and interleukin-2-activated lymphocytes. Annual Review of Immunology, 4: 681-709, 1986

31. S. A. Rosenberg, J. C. Yang, S. L. Topalian, D. J. Schwartzentruber, J. S. Weber, D. R. Parkinson, C. A. Seipp, J. H. Einhorn and D. E. White. Treatment of 283 consecutive patients with metastatic melanoma or renal cell cancer using high-dose bolus interleukin 2. JAMA, 271(12): 907-913, 1994

32. M. Rosenstein, S. E. Ettinghousen and S. A. Rosenberg. Extravasion of intravascular fluid mediated by the systemic administration of recombinant interleukin 2. Journal of Immunology, 137(5): 1735-1742, Sep 1986

33. Douglas J. Schwartzentruber. In Vitro predictors of clinical response in patients receiving interleukin-2-based immunotherapy. Current Opinion in Oncology, 5: 1055-1058, 1993

34. Abdolkarim Sohrabi, John Sandoz, John S. Spratt and Hiram C. Polk. Recurrence of breast cancer: Obesity, tumor size, and axillary lymph node metastases. JAMA, 244(3): 264-265, July 1980

35. E. Tartour, J. Y. Blay, T. Dorval, B. Escudier, V. Mosseri, J. Y. Douillard, L. Deneux, I. Gorin, S. Negrier, C. Mathiot, P. Pouillart and W. H. Fridman. Predictors of clinical response to interleukin-2-based immunotherapy in melanoma patients: a french multiinstitutional study. Journal of Clinical Oncology, 14(5): 1697-1703, 1996

36. Hensin Tsao, A. Benedict Cosimi, and Arthur J. Sober. Ultra-late recurrence (15 years or longer) of cutaneous melanoma. Cancer, 79(12): 2361-2370, 1997

37. Helen Vodopick, Elizabeth M. Rupp, C. Lowell Edwards, Francis A. Goswitz and John J. Beauchamp. Spontaneous cyclic leukocytosis and thrombocytosis in chronic granulocytic leukemia. The New England Journal of Medicine, 286(6): 284-290, Feb 1972 